Analytical approach for design of blazed dielectric gratings for light trapping in solar cells

This article has been downloaded from IOPscience. Please scroll down to see the full text article.

2011 J. Phys. D: Appl. Phys. 44055103

(http://iopscience.iop.org/0022-3727/44/5/055103)

View the table of contents for this issue, or go to the journal homepage for more

Download details:

IP Address: 150.203.162.16

The article was downloaded on 22/07/2011 at 07:07

Please note that terms and conditions apply. 


\title{
Analytical approach for design of blazed dielectric gratings for light trapping in solar cells
}

\author{
S Mokkapati, F J Beck and K R Catchpole \\ Centre for Sustainable Energy Systems, College of Engineering and Computer Science, The Australian \\ National University, Canberra, 0200, Australia \\ E-mail: sudha.mokkapati@anu.edu.au
}

Received 30 July 2010, in final form 2 November 2010

Published 17 January 2011

Online at stacks.iop.org/JPhysD/44/055103

\begin{abstract}
An analytical approach based on the scalar diffraction theory is presented for design of blazed diffraction gratings for maximizing the light trapping in solar cells. The model provides a conceptual insight into the behaviour of blazed structures. The predictions of the analytical model are checked against numerical results obtained using the commercial FDTD modelling software. Within the limits of the scalar diffraction theory, this analytical approach can be used to design gratings with arbitrary shapes.
\end{abstract}

(Some figures in this article are in colour only in the electronic version)

\begin{abstract}
A significant challenge facing the current photovoltaics industry is to bring down the cost of solar electricity to a level comparable to electricity generated from conventional sources such as fossil fuels. A substantial reduction in materials costs, and hence in the cost of electricity generated, can be achieved by developing thin film solar cells with an active layer thickness of a few micrometres, deposited on low-cost substrates. Because of the lower active region thickness the material quality required is also much lower than in waferbased cells, reducing the material costs further. However, a thin active layer means reduced light absorption, especially near the band edge of silicon ( $\mathrm{Si}$ ). In order to maximize light absorption efficiency it is essential to increase the optical thickness of the active layer by trapping light inside the active material.
\end{abstract}

Light trapping in thin solar cells can be achieved by directing light at large angles (outside the escape cone) into the absorbing layer using metal/dielectric scatterers or diffraction gratings [1-7]. For light trapping using diffraction gratings, light should be efficiently coupled into diffraction orders propagating outside the escape cone of Si. The light trapping characteristics of rectangular diffraction gratings have been extensively studied using the modal expansion method [8], proposed by Botten et al [9]. However, it has been proposed that symmetric grating structures such as rectangular gratings may not be the most efficient structures for light trapping $[4,5]$. The light-trapping efficiency of symmetric gratings is limited by coupling of diffracted light, after reflection from the opposite surface of the solar cell, to the outgoing zero-order wave. By employing asymmetric gratings, the coupling efficiency of diffracted light, reflected back from the opposite surface of the solar cell to the outgoing wave can be significantly reduced thereby increasing the light-trapping efficiency of the diffraction grating.

Unlike for rectangular diffraction gratings, there has been no simple analytical approach to date to design the blazed diffraction gratings for light-trapping applications. Even though it has been demonstrated, for a specific case [5], that blazed gratings can provide better light trapping than symmetric, rectangular groove grating structures, there has been no report on techniques to optimize the performance of blazed gratings. In this paper, we introduce a simple analytical approach for the design of blazed diffraction structures to maximize their light-trapping efficiency. The technique is applicable only for gratings with periods larger than the wavelength of the incident light, as it is based on the scalar diffraction theory $[10,11]$. We determine the transmission function for the periodic arrangement of blazed structures and compute the corresponding far-field diffraction pattern by applying a Fourier transform [12]. The model provides a conceptual understanding of the behaviour of the blazed structures. We show that the results of the analytical modelling agree well with the numerical 


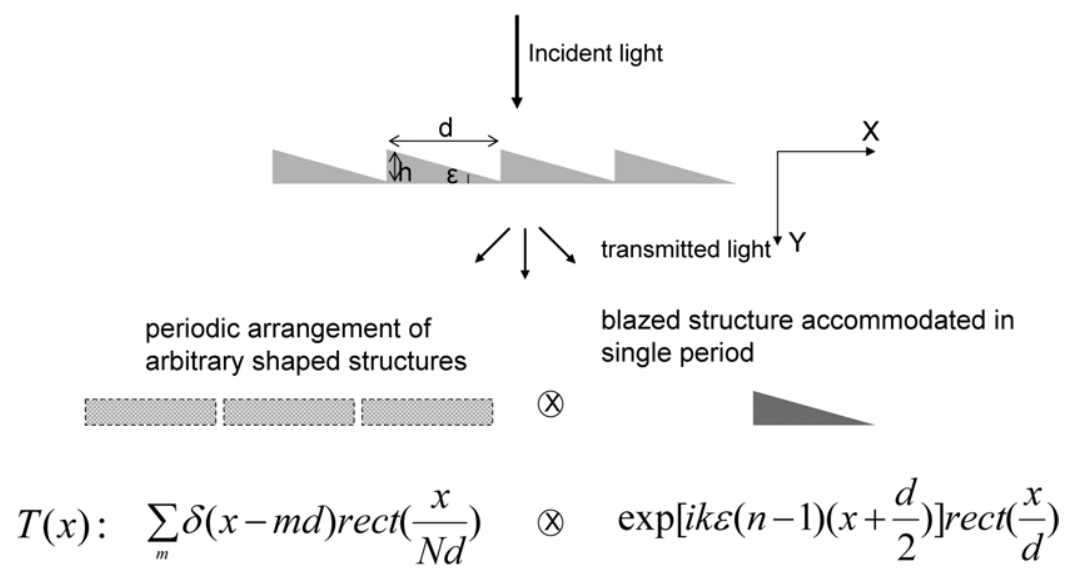

Figure 1. The blazed grating structure considered for demonstration of our analytical approach. The transmission function of the blazed grating is represented as the convolution of the transmission function of a periodic structure with periodicity $d$, and a blazed component with base $d$ and height $h . N$ is the number of periods considered for calculations and $n$ is the refractive index of the grating material. The expressions for the transmission functions are indicated below the corresponding structure.

results obtained using FDTD modelling. The proposed analytical modelling can be used to design and analyse various diffractive elements for light-trapping applications, without the need for the time and resource intensive numerical modelling/simulation techniques. And unlike the numerical modelling techniques that can only determine the response of the gratings, our analytical approach provides a phenomenological understanding of the physical concepts dictating the response of the gratings. In this paper, we illustrate the approach using blazed gratings, but it can also be used to design periodic structures with arbitrary shapes, as long as the period is larger than the wavelength of incident light. Key advantages of relatively large period diffraction gratings is that they are less wavelength sensitive, which is important in achieving light trapping for thin films which are weakly absorbing over a broad wavelength range. Relatively larger periods would also be expected to provide better coupling over a range of incident angles over the day, since small periods can only couple to normally incident light. The periods considered here are small enough to be able to be implemented in cells with few micrometre thick absorbing layers such as c-Si or a-Si/c-Si tandem thin-film cells.

The structure we consider to demonstrate our approach is illustrated in figure 1 . We consider a periodic arrangement of $N$ right-angled prisms with a base dimension of $d$ and height $h$ in air. $N$ has to be a large number for light-trapping applications. The total transmission function of the blazed grating $T(x)$ is expressed as the convolution of transmission function of a periodic arrangement (irrespective of the structure within the period) with a period $d, T_{\text {periodic }}(x)$, and that of a right-angled prism with base $d$ and height $h, T_{\text {prism }}(x)$. The periodic transmission function is 1 at integral multiples of $d$ and 0 elsewhere over a spatial range $N d$ and is expressed as [12]

$$
T_{\text {periodic }}(x)=\sum_{m} \delta(x-m d) \operatorname{rect}\left(\frac{x}{N d}\right),
$$

where $\operatorname{rect}(x)$ represents a rectangle function and is defined as $\operatorname{rect}(x)=1$ if $|x|<1 / 2$ and 0 elsewhere. Each individual right-angled prism has a transmission of 1 over a width $d$, but introduces a position $(x)$ dependent phase shift into the transmitted light. Thus the transmission function of the rightangled prism is expressed as [12]

$$
T_{\text {prism }}(x)=\exp \left[\mathrm{i} k \varepsilon(n-1)\left(x+\frac{d}{2}\right)\right] \operatorname{rect}\left(\frac{x}{d}\right),
$$

where the exponential term represents the phase function introduced by the prism, $k$ is the wave-vector of the incident light, $n$ is the refractive index of the grating material and the prism angle, and $\varepsilon$ is defined as $\tan ^{-1}(h / d)$.

The far-field light distribution resulting from the grating can now be evaluated using the Fourier transform of the total transmission function [11]. According to the convolution theorem, the Fourier transform of the convolution of two functions is the product of the Fourier transforms of the individual functions. Hence, the transmitted far-field light distribution due to the grating can now be evaluated from the Fourier transforms of the individual transmission functions represented in equations (1) and (2). Hence the normalized (to the maximum intensity) far-field intensity distribution resulting from transmission through the blazed grating structure becomes $[11,12]$

$$
I(v)=I_{\text {periodic }}(v) I_{\text {prism }}(v),
$$

where $I_{\text {periodic }}(v)$ is the Fourier transform of $T_{\text {periodic }}(x)$ and $I_{\text {prism }}(v)$ is the Fourier transform of $T_{\text {prism }}(x) . I_{\text {periodic }}(v)$ and $I_{\text {prism }}(v)$ are given by

$$
I_{\text {periodic }}(v)=\sum_{m} \sin c^{2}\left[v-\frac{m}{d}\right] N d
$$

and

$$
I_{\text {prism }}(v)=\sin c^{2}\left\{\left[v-\varepsilon \frac{n-1}{\lambda}\right] d\right\},
$$

where the $\sin c$ function is defined as $\sin c(x)=\sin (\pi x) / \pi x$, $v$ is the position of observation in the Fourier plane and $\lambda$ is the wavelength of the incident light.

To gain a conceptual understanding of the effect of varying grating parameters on the intensity distribution of the light 
$\mathrm{h}: 50 \mathrm{~nm}$ and $\lambda: 800 \mathrm{~nm}$

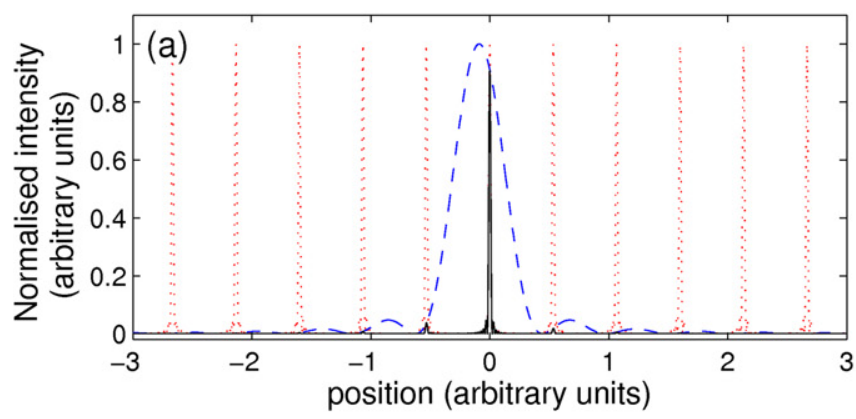

$\mathrm{h}: 300 \mathrm{~nm}$ and $\lambda: 800 \mathrm{~nm}$

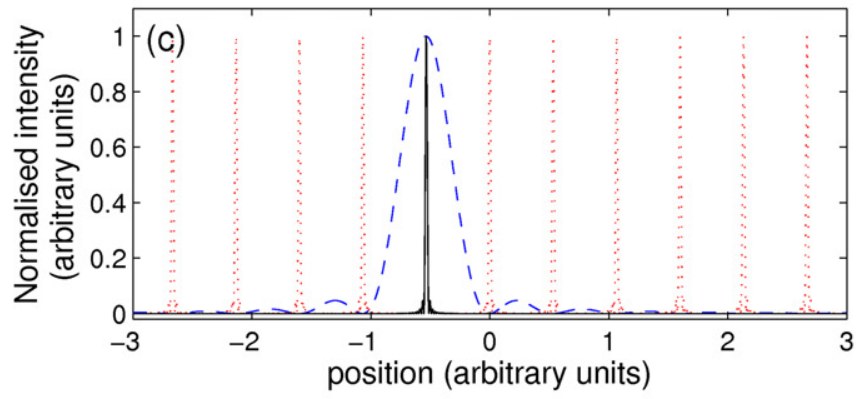

$\mathrm{h}: 150 \mathrm{~nm}$ and $\lambda: 800 \mathrm{~nm}$

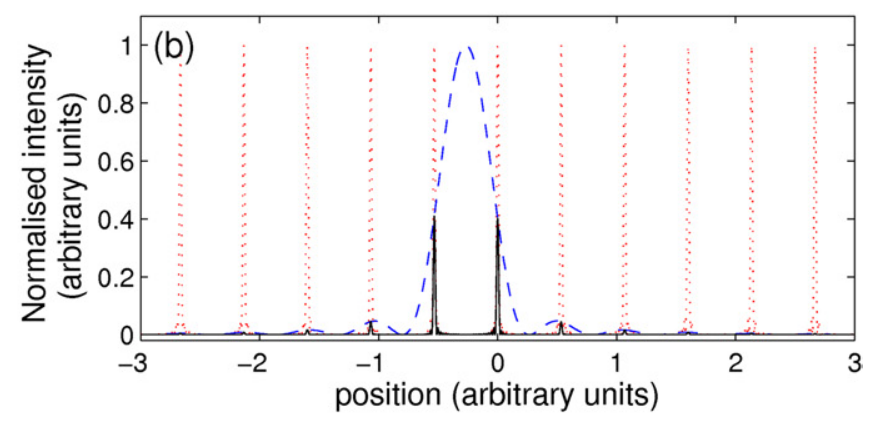

$\mathrm{h}: 500 \mathrm{~nm}$ and $\lambda: 800 \mathrm{~nm}$

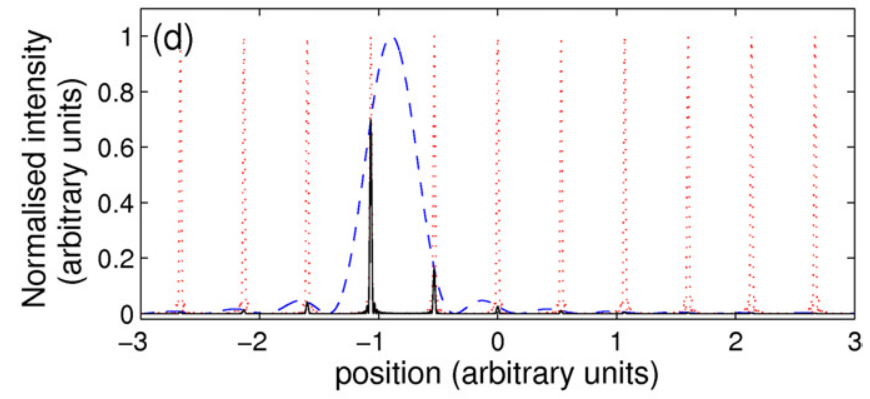

$\mathrm{h}: 600 \mathrm{~nm}$ and $\lambda: 800 \mathrm{~nm}$

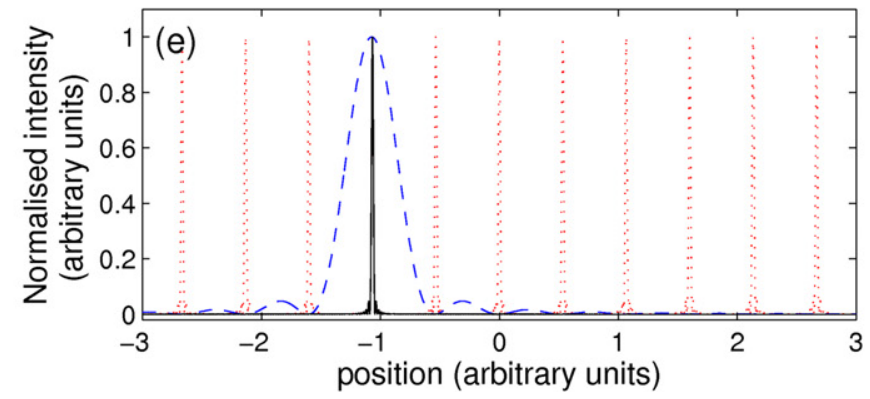

Figure 2. Normalized transmitted intensity distribution of a blazed Si grating with a period, $d 1500 \mathrm{~nm}$. The intensity distribution of the periodic arrangement (obtained using equation (4)) is represented in red, while the intensity distribution from a single prism (obtained using equation (5)) is represented in blue and the net intensity (evaluated using equation (3)) is represented in black. The wavelength of the incident light is fixed at $800 \mathrm{~nm}$ and the grating height is (a) $50 \mathrm{~nm},(b) 150 \mathrm{~nm},(c) 300 \mathrm{~nm},(d) 500 \mathrm{~nm}$ or (e) $600 \mathrm{~nm}$.

transmitted from the grating, we look at the effect of varying the height $h$ of a blazed Si grating on a Si substrate for a fixed period of $1500 \mathrm{~nm}$ and an incident wavelength of $800 \mathrm{~nm}$. The far-field intensity distribution from the blazed grating, along with the distribution for the periodic arrangement and a single prism, is shown in figure 2 for $h=50,150,300,500$ or $600 \mathrm{~nm}$. The propagation-angle-dependent intensity distribution data can be obtained from the position-dependent data presented in figure 2 by determining the propagation angle corresponding to each diffraction order using the one-dimensional grating equation. For $h=50 \mathrm{~nm}$, the intensity distribution of a single prism is concentrated near the 0th order transmission peak of the periodic structure. Hence the net transmission intensity of the blazed grating is mostly concentrated in 0th or principal diffraction order, with only a very small fraction of light coupled into +1 and -1 diffraction orders. When the grating height is increased to $150 \mathrm{~nm}$, the transmission peak from the single prism is centred between the 0 th and -1 diffraction orders of the periodic structure, resulting in equal intensity distribution among the 0 and -1 diffraction orders for the blazed grating. Most of the transmitted light is concentrated in these two diffraction orders, with only a small fraction of light coupled into +1 and -2 diffraction orders, as the positions of these diffraction orders coincide with the position of secondary maxima of the prism diffraction pattern. By increasing the grating height to $300 \mathrm{~nm}$, the principal maxima of the prism diffraction pattern coincides exactly with the position of -1 diffraction order of the periodic structure and the position of the rest of the diffraction orders of the periodic structure coincide with the minima in the diffraction pattern of the prism. As a result, all the light transmitted from the blazed grating is coupled into -1 diffraction order. By increasing the grating height to $500 \mathrm{~nm}$, the principal maxima in the prism diffraction pattern moves closer to -2 diffraction order of the periodic structure. In this case, the light transmitted from the blazed grating is mostly coupled to -1 and -2 diffraction orders, with a larger fraction being coupled into the -2 diffraction order.

For light-trapping applications, a large fraction of the light transmitted from the blazed grating should be coupled 
into diffraction orders propagating outside the escape cone of Si. For incident light with wavelength of $800 \mathrm{~nm}$ (at $800 \mathrm{~nm}$, refractive index of $\mathrm{Si}$ is 3.67), the half-angle for the escape cone calculated using Snell's law is $15.8^{\circ}$. For a period of $1500 \mathrm{~nm}$ (the parameter chosen figure 2), \pm 1 diffraction orders propagate at an angle of $8.4^{\circ}$ and \pm 2 diffraction orders propagate at an angle of $16.9^{\circ}$ with respect to the surface normal. So light should be coupled into \pm 2 or higher diffraction orders for getting trapped inside $\mathrm{Si}$. For a grating height of $500 \mathrm{~nm}$, a large fraction of the transmitted light is trapped inside Si. For all the transmitted light to be trapped, a grating height of at least $600 \mathrm{~nm}$ should be chosen, as for this condition the principal maxima in the diffraction pattern of the prism coincides with the position of -2 diffraction order of the periodic structure. All the transmitted light of wavelength $800 \mathrm{~nm}$ will be trapped for grating heights larger than $600 \mathrm{~nm}$.

While a grating height of $600 \mathrm{~nm}$ is sufficient to trap incident light of wavelength $800 \mathrm{~nm}$ inside the solar cell, for light-trapping applications, gratings have to be optimized for trapping light over a broad spectral range. Light trapping becomes critical for light in the long wavelength region $(>800 \mathrm{~nm})$ of the solar spectrum. From figure 2, we can infer that for efficient light trapping, grating height larger than $600 \mathrm{~nm}$ should be used. By employing grating height $>600 \mathrm{~nm}$, a larger fraction of light of wavelength $>800 \mathrm{~nm}$ will be coupled to diffraction orders propagating outside the escape cone inside $\mathrm{Si}$. The increase in percentage absorption over the solar spectrum can be used to quantify the efficiency of a grating for light-trapping applications. We use a commercially available package from Lumerical [13] to determine the percentage absorption over the solar spectrum for different grating parameters through FDTD simulations. A planar Si film of thickness $1 \mu \mathrm{m}$ absorbs $13.3 \%$ of the solar spectrum. By using a blazed, non-absorbing diffraction grating of period $1500 \mathrm{~nm}$ and height $50 \mathrm{~nm}$ on the front, the absorption in the same thickness of Si is increased to $14.7 \%$, while by employing a grating of height $900 \mathrm{~nm}$, the absorption is increased to $38 \%$. These results are consistent with the prediction of the analytical modelling that a grating height of at least $600 \mathrm{~nm}$ is required for efficient light trapping in the wavelength range $>800 \mathrm{~nm}$. The above numbers indicate that the analytical model is a simple and efficient means of determining the optimal grating parameters for light-trapping applications.

We now compare the results of the analytical modelling with results obtained using FDTD simulations (using a commercially available package from Lumerical [13]) to check the validity of the technique. FDTD gives numerical results for the field intensities in the region of interest, by solving the complete set of Maxwell's equations. Figures 3(a), (b) and $(c)$ show the fraction of light transmitted through a $\mathrm{Si}$ grating that is coupled into $0,-1$ and -2 diffraction orders in a Si substrate at different wavelengths, as the grating height is varied. The grating has a period of $1500 \mathrm{~nm}$. The data represented in the contour plots on the top row are obtained using FDTD. The data presented in the contour plots on the bottom row in figures $3(a)-(c)$ are obtained using the analytical modelling. Figure $3(d)$ shows line plots of the data obtained using FDTD and the analytical modelling for the fraction of light coupled into various diffraction orders, as a function of wavelength, for a fixed grating height of $100 \mathrm{~nm}$. Figure 3(e) shows the line plots of the data obtained using FDTD and the analytical modelling for the fraction of light coupled into various diffraction orders, as a function of grating height, for a fixed wavelength of $700 \mathrm{~nm}$.

From the top row of contour plots (figures 3(a), (b) and $(c)$ ), for small grating heights, FDTD results show that most of the diffracted light is coupled into the 0th diffraction order. As the grating height increases, the fraction of light coupled into the -1 diffraction order increases gradually, reaches a maximum and then starts to decrease again. As the light coupled into -1 diffraction order begins to fall, the fraction of light coupled into the -2 diffraction order begins to increase. This trend agrees very well with the predictions of the analytical modelling (bottom row of figures $3(a)-(c)$ ). As also can be seen from the line plots in figures $3(d)$ and $(e)$, the fraction of light coupled into various diffraction orders calculated using the analytical modelling matches well with the data from FDTD.

We would like to note that the analytical modelling presented here does not take into account the wavelength scale edge effects occurring in the grating. Also, the model does not account for index matching between the grating material and the substrate/superstrate. As can be observed from figures 3(d) and $(e)$, the variation between the Lumerical data and the analytical modelling data is maximum for small wavelengths or for larger angle of propagation of light because of the above two effects. For example, for a grating height of $100 \mathrm{~nm}$, at a wavelength of $500 \mathrm{~nm}$ (figure $3(d)$ ), the fraction of light coupled into -1 diffraction order, as predicted by the analytical modelling exceeds the value determined by Lumerical by $17 \%$. For a grating height of $500 \mathrm{~nm}$ and an incident light wavelength of $700 \mathrm{~nm}$ (figure $3(e)$ ), the fraction of light coupled into -2 diffraction order as predicted by the analytical modelling exceeds the value determined by Lumerical by $40 \%$. The above two effects should also be accounted for in order to get more accurate results. However, the approach outlined here does provide us with a conceptual understanding of the phenomena determining the light coupling into various diffraction orders from a blazed grating.

We now demonstrate with an example how the analytical modelling can be used to design structures for specific applications. The dashed blue line in figure 4(a) shows the diffraction intensity, for an incident wavelength of $800 \mathrm{~nm}$, from an asymmetric prism (structure considered thus far, and illustrated in figure 1) of base, $d=1500 \mathrm{~nm}$ and height, $h=$ $200 \mathrm{~nm}$. The diffraction peak is positioned between the 0th and -1 diffraction orders arising from a periodic arrangement with a periodicity $1500 \mathrm{~nm}$. Hence the diffracted light from the asymmetric blazed grating is coupled mostly into the 0th and -1 diffracted orders. $\sim 70 \%$ of transmitted light is coupled into -1 diffraction order and $\sim 20 \%$ of transmitted light is coupled into 0th diffraction order, as shown by the solid blue lines in figure 4(a). These results are in agreement with results obtained using Lumerical. Lumerical simulations show that for the above grating parameters, $\sim 50 \%$ of transmitted light is coupled to -1 diffraction order and $25 \%$ of the transmitted 

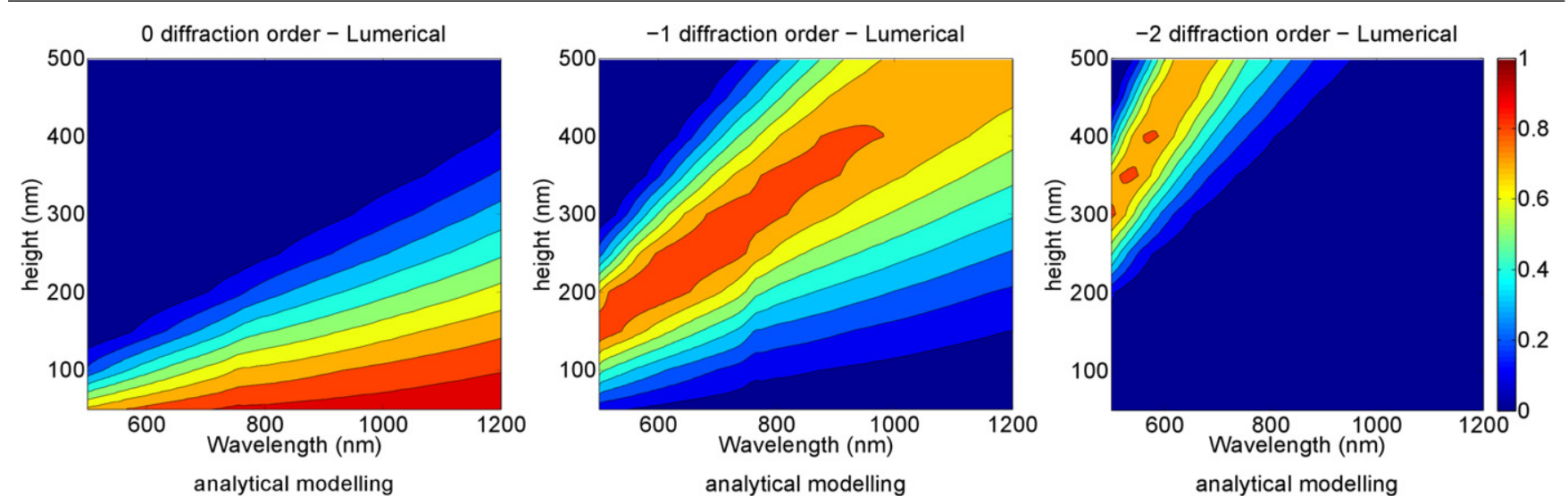

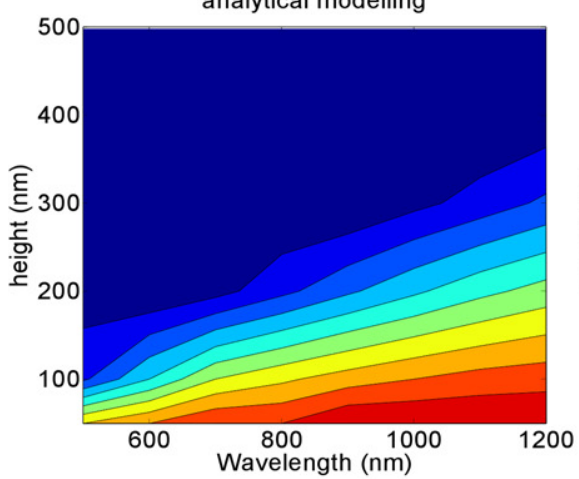

(a)

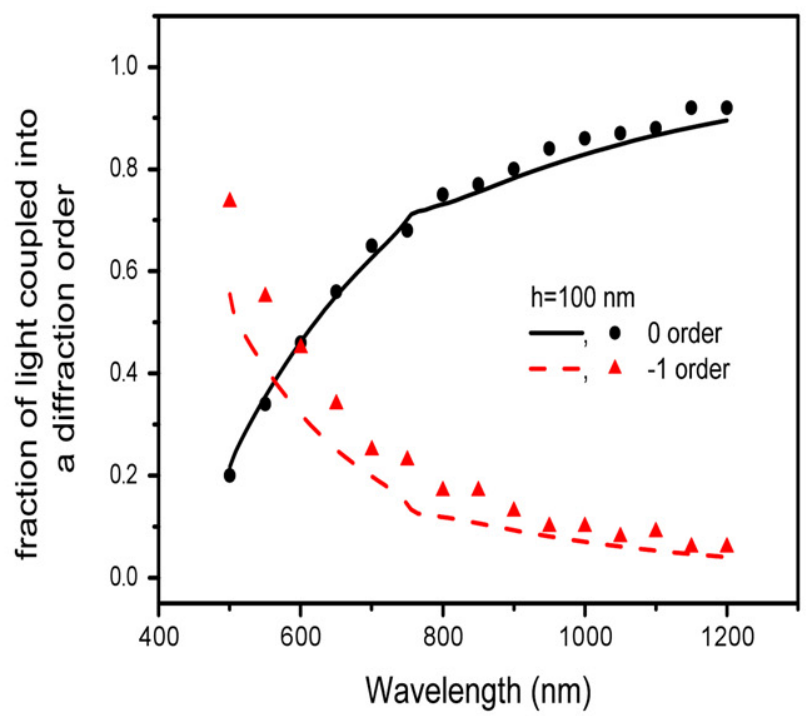

(d)

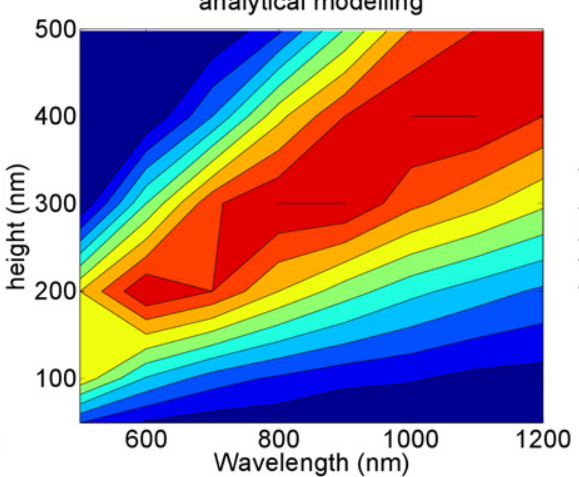

(b)

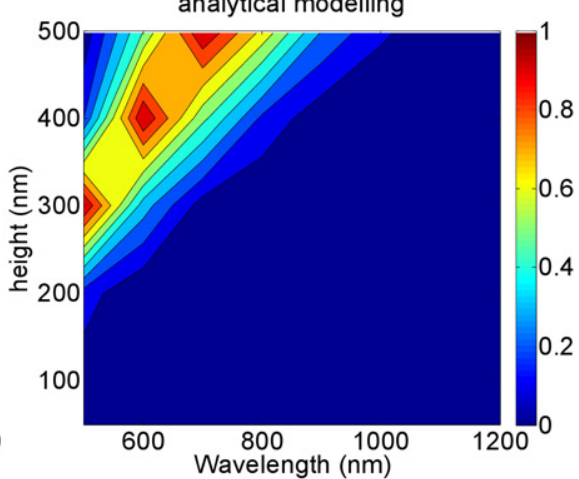

(c)

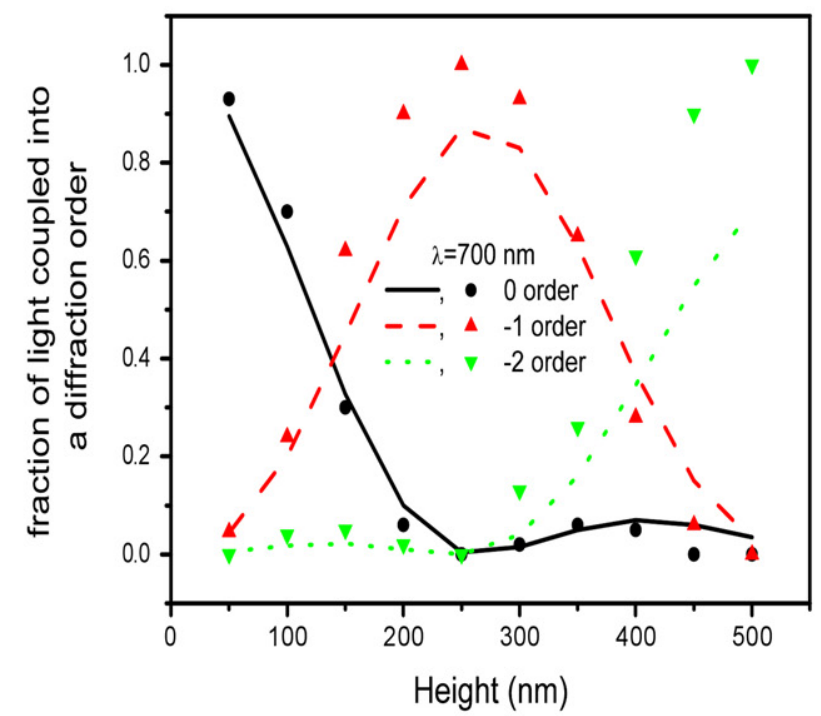

(e)

Figure 3. Fraction of transmitted light coupled into $(a) 0,(b)-1$ and $(c)-2$ diffraction orders. The contour plots on the top row show data obtained using numerical modelling (FDTD) and the contour plots on the bottom row show data obtained using the analytical model. The data are for Si gratings on Si substrates. Grating period is $1500 \mathrm{~nm}$ and the grating height is varied between 50 and $500 \mathrm{~nm}$. $(d)$ and $(e)$ show line plots representing selected cross-sections of $(a)$ and $(b)$ for ease of comparison. $(d)$ shows fraction of light coupled into 0 and -1 diffraction orders for a grating height of $100 \mathrm{~nm}$ as the wavelength of incident light is varied. $(e)$ shows fraction of light coupled into $0,-1$ and -2 diffraction orders at a wavelength of $700 \mathrm{~nm}$ as the grating height is varied. The lines are from numerical modelling and the points are from analytical modelling.

light is coupled into 0th diffraction order. The difference between the values predicted by the analytical modelling and by Lumerical simulations is again attributed to the causes described earlier. As discussed earlier, for light trapping, the diffracted light needs to be coupled into higher order $(> \pm 2)$ diffraction orders. One approach to achieve this would be to increase the grating (prism) height such that the diffraction peak from a single prism is positioned closer to a higher order diffraction mode from the periodic arrangement, as illustrated in figure 2. Alternatively, keeping the grating height the same, the structure within a single period can be modified, as shown in the inset of figure $4(b)$, to direct light into higher 


\section{$\mathrm{h}: 200 \mathrm{~nm}$ and $\lambda: 800 \mathrm{~nm}$}
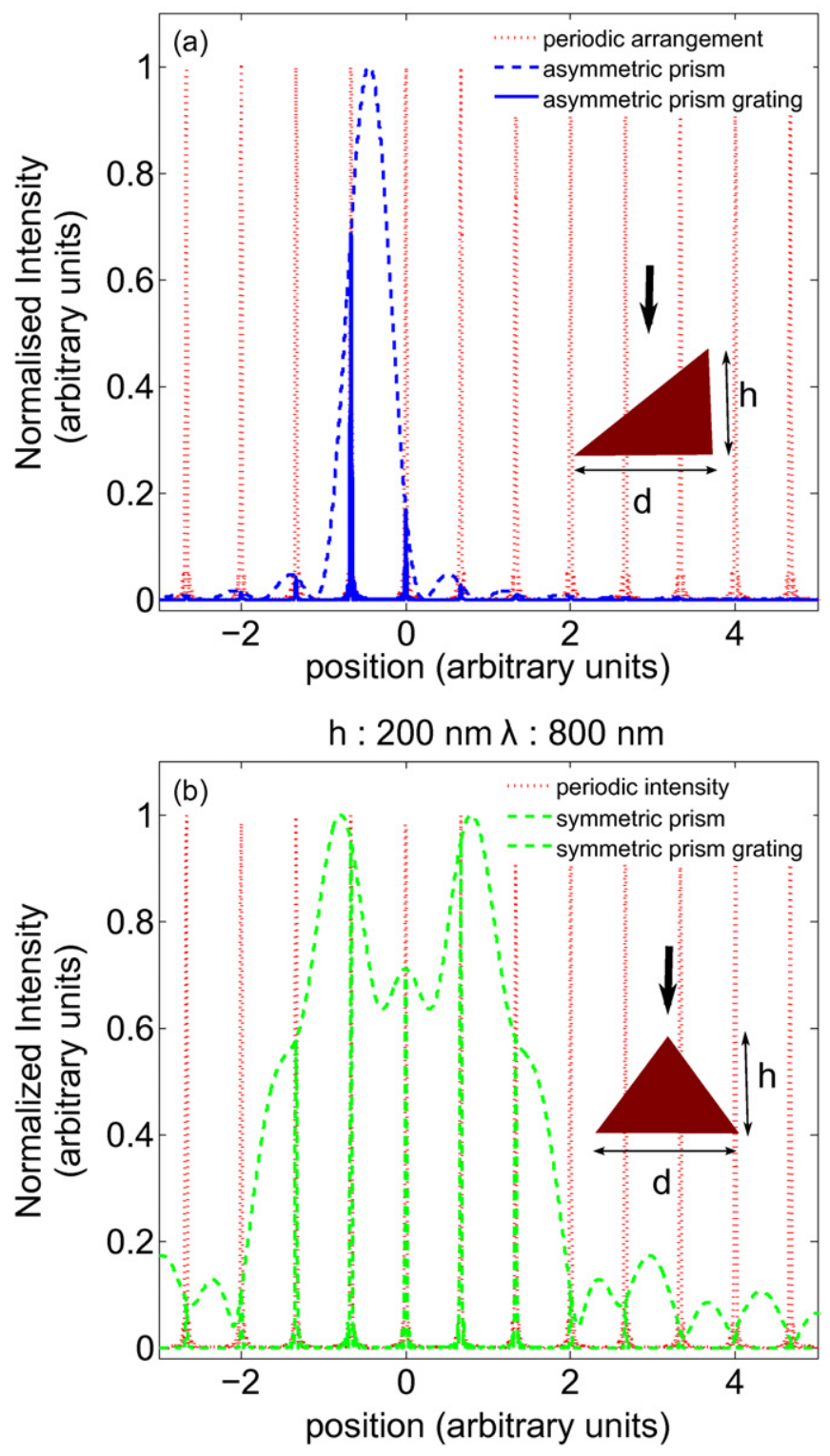

Figure 4. Normalized transmitted intensity distribution for $(a)$ blazed and (b) symmetric Si gratings with a period, d $1500 \mathrm{~nm}$. The intensity distribution of the periodic arrangement is represented in red dotted lines. The blue dashed line represents the diffraction intensity from a single linearly blazed, asymmetric prism; the green dashed line represents the diffraction intensity from a symmetrical prism. The solid lines represent the transmitted intensities from the respective gratings.

order diffraction modes. The transmission function of this symmetric prism grating within a single period is now the convolution of the transmission functions of two asymmetric prisms with base dimension $d / 2$, and height $h$ and is given by

$$
\begin{aligned}
& T_{\text {symm-prism }}(x)=\exp [\mathrm{i} k \varepsilon(n-1) x]\left[\operatorname{rect}\left(\frac{2 x}{d}-\frac{1}{2}\right)\right] \\
& \otimes \exp [\mathrm{i} k \varepsilon(1-n)]\left[\operatorname{rect}\left(\frac{2 x}{d}+\frac{1}{2}\right)\right] .
\end{aligned}
$$

The narrower transmission function for the modified structure indicates that the far-field diffraction intensity, which is the Fourier transform of $T_{\text {symm-prism }}(x)$, will be broader compared with that of a prism with base dimension $d$. Because of the way the two asymmetric prisms are positioned within a period, each of them directs light onto opposite sides of the Oth diffraction order, as shown by the dotted green line in figure $4(b)$. The light transmitted through the symmetric prism grating is now coupled into higher order diffraction modes, as shown by the solid green lines in figure $4(b)$. The analytical model predicts that $\sim 20 \%$ of transmitted light is coupled into 0 th diffraction order, $\sim 50 \%$ of transmitted light is coupled into \pm 1 diffraction orders and $\sim 30 \%$ of transmitted light is coupled into \pm 2 diffraction orders. Lumerical simulations show that $\sim 20 \%$ of transmitted light is coupled into 0th diffraction order and $\sim 80 \%$ of transmitted light is coupled into the \pm 1 diffraction orders. Again, we attribute the differences between the values predicted by the analytical model and Lumerical to the causes described earlier. This example illustrates how the analytical modelling can be used to design diffraction gratings to achieve a desired diffraction pattern.

In summary, we have presented a simple analytical modelling technique to design diffraction gratings for lighttrapping applications in solar cells. The model is applicable (in the limits of the scalar diffraction theory) to gratings of any shape as long as the transmission function for a single period can be defined. The results of the analytical model agree very well with that of numerical results obtained using FDTD. Unlike numerical techniques that can be time- and resource-intensive, this simple analytical approach provides a phenomenological understanding of the behaviour of blazed structures.

\section{Acknowledgments}

The authors thank the Australian Research Council for financial support.

\section{References}

[1] Zhao L, Zuo Y H, Zhou C L, Li H L, Diao H W and Wang W J 2010 Sol. Energy 84 110-5

[2] Mutitu J G, Shi S, Chen C, Creazzo T, Barnett A, Honsberg C and Prather D W 2008 Opt. Express 16 15238-48

[3] Matheu P, Lim S H, Derkacs D, McPheeters C and Yu E T 2008 Appl. Phys. Lett. 93113108

[4] Morf R H, Kiess H and Heine C 1997 Diffractive Optics for Industrial and Commercial Applications (Berlin: Akademie)

[5] Heine C and Morf R H 1995 Appl. Opt. 34 2476-82

[6] Beck F J, Mokkapati S, Polman A and Catchpole K R 2010 Appl. Phys. Lett. 96033113

[7] Atwater H A and Polman A 2010 Nature Mater. 9 205-13

[8] Catchpole K R 2007 J. Appl. Phys. 102013102

[9] Botten I C, Craig M S, McPhedran R C, Adams J L and Andrewartha J R 1981 Opt. Acta: Int. J. Opt. 28 413-28

[10] Born M and Wolf E Principles of Optics 1999 7th edn (Cambridge: Cambridge University Press)

[11] Goodman J W Introduction to Fourier Optics 2005 (New York: McGraw-Hill)

[12] Sinzinger S and Testorf M 1995 Appl. Opt. 34 5970-6

[13] Lumerical FDTD Solutions, http://www.lumerical.com 